\title{
Recombinant subunits of SARS-CoV-2 spike protein as vaccine candidates to elicit neutralizing antibodies
}

Faezeh Noorabad ghahroodi

Tarbiat Modarres University

Saeed Khalili

Shahid Rajaee Teacher Training University

Mohammad Javad Rasaee ( $\nabla$ rasaee_m@modares.ac.ir)

Tarbiat Modarres University

\section{Research Article}

Keywords: SARS-COV-2, spike, vaccine, neutralizing antibody, in silico,

Posted Date: April 28th, 2021

DOI: https://doi.org/10.21203/rs.3.rs-414366/v1

License: (c) (i) This work is licensed under a Creative Commons Attribution 4.0 International License.

Read Full License 


\section{Abstract}

The spike protein has been reported as one of the most critical targets for vaccine design strategies against the SARS-COV-2 infection. Hence, we have designed, produced, and evaluated the potential use of recombinant proteins derived from spike protein as vaccine candidates capable of neutralizing SARSCOV-2 virus.

In silico tools were used to design spike-based subunit recombinant proteins (P1, P2, and P3). These proteins were checked for their ability to be identified by the anti-SARS-COV-2 antibodies by exposing them to Covid-19 serum samples. The proteins were then injected into mice and rabbits and the antibody titers were measured for 170 days. The virus neutralization test (VNT) was performed to analyze the obtained antibodies for their neutralization efficiency.

The antibodies that existed in the serum of COVID-19 patients have identified the designed proteins. The anti-spike antibody titer was increased in the animals injected with recombinant proteins. The VNT results revealed that the produced antibodies could neutralize the cultured live virus. The long-lasting antibody titers (130 and 170 days for rabbit and mouse groups) indicated the elicitation of a strong immune response by the recombinant proteins.

Subunit vaccines could also be considered as robust tools for effective vaccination against COVID-19. Using a combination of in silico, in vitro, and in vivo experiments, it was shown that the injection of spikebased recombinant proteins could stimulate long-lasting and neutralizing antibody responses. Further evaluation of the recombinant proteins examined in our studies in higher primates and human would elucidate their true potentials.

\section{Introduction}

The outbreak of the new coronavirus (SARS-CoV-2) is considered as the third viral disease of the coronavirus family in the 21st century [1]. This virus rapidly spreads through the inhalation of respiratory aerosols. Clinical symptoms vary from no symptoms to highly significant clinical symptoms such as severe respiratory syndrome $[2,3]$. Primary transmitters of SARS-CoV-2 would be the patients with no signs and symptoms [4]. Since the beginning of the Covid-19 outbreak, many strategies and approaches have been proposed to fight against it. Disinfection of passages, quarantine, social isolation, public safety, drugs, and finally, vaccines were the leading solutions offered by health care providers and specialists [5]. Contemporary, various treatment strategies and vaccine production approaches are on the agenda of all countries. Oxygen therapy, antibiotics, antifungals, antivirals, glucocorticoids, and immunoglobulins are among the practiced treatment protocols $[2,6]$. Coming to grips with the life cycle and pathogenesis mechanism of the SARS-CoV-2 would bring about insights into the proper strategies to develop vaccines.

SARS-CoV-2 is a single-stranded sense RNA virus. It belongs to the Sarbecovirus subgroup of the Betacoronavirus group and the subfamily of the Orthomixovirinae. Coronaviruses are divided into four 
groups, including $\delta, \gamma, a$, and $\beta \operatorname{CoV}[7,8]$. The size of the viral genome is between 26 to $32 \mathrm{~kb}$, which is considered as one of the largest RNA viruses [9]. The diameter of these viruses is about 60 to $140 \mathrm{~nm}$ [10]. Two-thirds of the viral RNA transcribes for the pp1a, pp1ab, and 16 non-structural proteins. The rest of the genome encodes for structural proteins [11]. The main structural protein includes the spike (S), nucleocapsid $(N)$, envelope $(E)$, and membrane $(M)$ proteins, which are encoded by the $3^{\prime}$ end of the viral genome $[11,12]$.

SARS-CoV-2 enters the host cells through the angiotensin-converting enzyme (ACE2) receptor [13, 14], which resides in the human lower respiratory tract. It is also known as the cellular receptor for SARS-COV $[15,16]$. The S glycoprotein on the surface of the SARS-CoV-2 can bind to the ACE2 receptor on the surface of human cells [17]. This protein is a trimmer protein that belongs to class 1 viral glycoproteins [18]. It has been reported that the $S$ proteins are the most important structural protein of the SARS-CoV-2 to enter the target cells. The S glycoprotein plays an essential role in viral binding, fusion, and entry into the host cell [19]. This protein has two significant subunits known as S1 and S2 subunits. The S1 subunit identifies the host cell, while the S2 subunit is responsible for the fusion of the virus to the host cells [2022]. The $\mathrm{N}$-terminal domain (NTD) and the receptor-binding domain (RBD) are the functional domains of the $\mathrm{S} 1$ subunit. The RBD encompasses a receptor-binding motif (RBM) that is conserved among most strains of coronaviruses [23]. The RBD sequence from the SARS-CoV shares $72 \%$ similarity with the RBD from SARS-CoV-2[24]. Prior studies have revealed that the RBD can form a tight interaction with ACE2 protein ,which initiates the infection process[25]. The interaction between the SARS-CoV-2 and the ACE2 receptor is mainly occurred, between the RBM from the $S$ protein and the $N$ terminus region of the ACE2. This interaction leads to endocytosis of the virus [25]. The interaction between the RBD and the ACE2 receptor stimulates structural changes in the $\mathrm{S} 2$ subunit. The exerted changes play an essential role in the fusion between the viral envelope and the host cell membrane. The S2 subunit of the S protein consists of several regions, including the membrane-anchoring region, the fusion peptide (FP), the heptad repeat (HR) 1, and the HR 2.[23] Inside the endosome, the S1 subunit would be cleaved off, and the FP would be exposed. The FP locates itself inside the host membrane. The $\mathrm{S} 2$ then bends over to bring HR1 and HR2 together. This causes membrane fusion and release of the viral genome in the cytoplasm of the host cell $[24,26,27]$.

According to WHO, 198 vaccines are under investigation worldwide at various stages. Various vaccine development strategies have been practiced in different settings preclinical and clinical trials, including the DNA, RNA, recombinant protein, viral vector, and the attenuated or deactivated vaccines. Safety considerations and the wide variety of antigen variants are potential challenges ahead of efficient vaccine development. The pivotal role of the S protein in the pathogenesis of the SARS-CoV-2 confirms that it can be the principal antigenic agent for stimulation of the host immune system and production of neutralizing antibodies[28]. Prior studies have shown that vaccines made from the $S$ protein can stimulate the immune system and induce humoral and cellular responses.

The ongoing studies regarding the design of SARS-CoV-2 vaccines are already focused on the S protein using different platforms. In light of these observations, we aimed to design vaccine candidates based on 
the mechanism of S protein action and bioinformatics analyses. In this regard, three antigenic regions of the $S$ protein were selected as vaccine candidates to elicit humoral immunization against SARS-CoV-2, which may induce neutralizing antibodies. The immunogenicity of these vaccine candidates was evaluated using in silico, in vitro, and in vivo studies.

\section{Methods}

All experimental protocols were approved by the Faculty of Medical Sciences Tarbiat Modarres University (TMU) and National Institute of Genetic Engineering and Biotechnology.

\subsection{Sequence retrieval}

The sequence of the S protein was retrieved from the NCBI database at https://www.ncbi.nlm.nih.gov/. The obtained sequence was used to perform a BLAST search at https://blast.ncbi.nlm.nih.gov/Blast.cgi. The PSI-BLAST (Position-Specific Iterated BLAST) tool of the protein BLAST was employed to find the highly similar protein sequences. Multiple sequence analysis was performed on the sequences obtained from the BLAST search. The potential glycosylation sites on the S protein were predicted using NetNGlyc1.0 software at http://www.cbs.dtu.dk/services/NetNGlyc/. The S protein sequence was also searched for the existence of a signal peptide using SignalP-5.0 software at http://www.cbs.dtu.dk/services/SignalP/.

\subsection{Sequence analyses of the selected regions}

The ProtParam software at https://web.expasy.org/protparam/ was used to predict the physicochemical properties of the selected regions. The potential glycosylation sites on the selected regions were predicted using NetNGlyc-1.0 software. The antigenicity of the selected sequences was predicted by Vaxijen-2.0 software at http://www.ddg-pharmfac.net/vaxijen/VaxiJen/VaxiJen.html. The allergenicity of the regions was predicted by Algpred software at http://crdd.osdd.net/raghava/algpred/. The toxicity of the selected regions was predicted by ToxinPred software at http://crdd.osdd.net/raghava/toxinpred/.

\subsection{Recombinant expression of the candidate vaccines}

The protein sequence of the selected regions was reverse transcribed to the DNA sequences by the EXPASY translate tool at http://web.expasy.org/translate/. The Jcat tool at http://www.jcat.de/ was employed to optimize the DNA sequences for high levels of protein expression (the E. coli codon usage bias was used for the optimization). The EcoR1 and Xhol restriction sites were selected to insert the designed genes within the pET28a expression vector. This design would grantee the expression of His tag sequence at the $\mathrm{N}$ terminus of the proteins. The final genes were ordered for chemical synthesis and subsequent sub-cloning by the GENERAY Biotechnology Company. The synthesized genes (within the pET28a expression vector) were transformed into E.coli BL21 (DE3) using the standard $\mathrm{CaCl}_{2}$ method. Colony PCR using the universal T7 primers was employed to confirm the transformation. The protein expression of the transformed cells was performed using the same method employed in our previous study [29]. The expression of the protein was optimized at different durations $(4 \mathrm{H}, 8 \mathrm{H}$, and $16 \mathrm{H})$, IPTG concentrations $(0.3,0.5,0.8$, and $1 \mathrm{mM})$, and temperatures $\left(18,25\right.$, and $\left.37^{\circ} \mathrm{C}\right)$. The total proteins of the 
expressed cultures were analyzed by SDS-PAGE in $15 \%(\mathrm{~W} / \mathrm{V})$ polyacrylamide gel. The gel was stained by standard Coomassie brilliant blue G-250 for $4 \mathrm{H}$ and de-stained with $45 \%$ methanol/10\% acetic acid solution.

\subsection{Protein purification and Western blotting}

Protein purification and Western blot analysis were performed using the protocol adapted from our previous study [29]. Briefly, the $\mathrm{Ni}^{+}-\mathrm{NTA}$ resin-packed columns (Qiagen, Germany) were used to purify the expressed proteins following the procedures provided by the manufacturer. The $\mathrm{pH}$ gradient of a lysis buffer was used to purify the protein. The SDS-PAGE (4\% stacking gel and $15 \%$ separating gel) was used to analyze the genes' expression and the purity of the eluted fractions. The purification fraction (with the $\mathrm{pH}$ of 5.2), which contained a single protein band of the recombinant protein, was selected for the following experiments. The protein content of the eluted fractions was measured using standard Bradford assay. Since the proteins formed inclusion bodies, they were denatured using standard denaturing conditions by $8 \mathrm{M}$ urea. The purified protein samples were finally dialyzed to remove the urea and increase the $\mathrm{pH}$ from 5.2 to 7.4. These changes prepare the conditions for protein renaturation. A standard Western blot analysis was performed to assess the yield of the purification step. In this regard, primarily, the proteins were resolved on the gel; after that, the proteins were transferred onto the nitrocellulose membrane (Whatman Schleicher and Schuell, Dassel, Germany). Ultimately, an anti-His tag antibody conjugated with horseradish peroxidase (HRP) was added to the nitrocellulose membrane.

\subsection{Circular Dichroism (CD) Spectroscopy}

The CD Spectroscopy study was performed in the far UV range (the wavelength range of 190-260 nm) (Far-UV-CD). This wavelength range is consistent with the absorption of peptide bonds. The study was performed with Jasco's J-810 spectropolarimeter. Each secondary structure creates a unique curve according to the rotation angle of its peptide bond. To achieve the experiment, $0.2 \mathrm{mg} / \mathrm{ml}$ of each protein was dissolved in $10 \mathrm{mM}$ phosphate buffer of different molarities of urea and then placed in a CD spectroscopy machine. The obtained spectra were corrected against PBS buffer and other molarities of urea base buffer $(0,1,2,4,6$, and 8$)$ as a control using a unique noise reduction software. The obtained data were analyzed at https://npsa-prabi.ibcp.fr/ to calculate the percentage of secondary structures.

\subsection{Animal immunization and production of polyclonal antibody}

The study conduction was adhered to the principles of the declaration of ARRIVE guidelines based on Ethical Committee Compliance of TMU and National Institute of Genetic Engineering and Biotechnology (code of ethics: 1399.015), furthermore all methods were carried out in accordance with relevant guidelines and regulations of TMU. The purified proteins were used for animal immunization. Three groups (2 rabbits per each group) of female New Zealand white rabbits (8 weeks old) and four groups (3 mice per each group) of BALB/c female mice (4-6 weeks old) (Razi Institute, Karaj, Iran) were used to elicit polyclonal antibodies. Each group of rabbits received one of the candidate vaccine proteins. There was a different mice group that received a mixture of all three antigens to check their combinatorial immunization ability. The rabbits were first injected (intra-muscularly into the large muscle of the rear legs) $350 \mu \mathrm{g}$ of each vaccine candidate protein along with the same volume of Freund's complete 
adjuvant. The booster injections were given at 15-day intervals with $350 \mu \mathrm{g}$ of each vaccine candidate protein mixed with incomplete Freund's adjuvant. The first bleeding was performed before the injections; bleedings were done after the third injection and repeated two more times at 15-day interval. The rabbit serum antibody titers were measured using the indirect ELISA method. To determine the antibody titer, briefly, $1 \mu \mathrm{g}$ of the purified proteins were coated on a 96-well immunoplate at $37^{\circ} \mathrm{C}$ overnight. Then, the coated wells were incubated with serially diluted serum (1:500, 1:1000, 1:2000, and 1:4000 in PBS). Afterward, the wells were incubated with 1:4000 diluted HRP-conjugated mouse anti-rabbit IgG (Thermo Scientific, MA, USA) for $90 \mathrm{~min}$ at $37^{\circ} \mathrm{C}$. Finally, $50 \mu \mathrm{l}$ of tetramethylbenzidine substrate reagent (BD Biosciences Pharmingen, CA, USA) was added to each well and incubated for $15 \mathrm{~min}$ at $37^{\circ} \mathrm{C} .50 \mu \mathrm{l}$ of $2 \mathrm{~N}$ of $\mathrm{HCl}$ was used to stop the color development. The absorbance was measured at $450 \mathrm{~nm}$. The mice were injected twice at 15-day interval. The bleeding was done before injections and 90 and 120 days after the last injection. The mice were injected intramuscularly with $50 \mu \mathrm{g}$ of each vaccine candidate protein along with the same volume of Freund's complete adjuvant. The booster injection was given after 15 days with $50 \mu \mathrm{g}$ of each vaccine candidate protein mixed with incomplete Freund's adjuvant. The fourth mice group was injected with $150 \mu \mathrm{g}$ of the three vaccine candidates $(50 \mu \mathrm{g}$ of each vaccine was mixed) mixed with incomplete Freund's adjuvant. To determine the antibody titer, the same indirect ELISA method was employed.

\subsection{Antibody purification}

Antibody purification for all serum samples was done using affinity chromatography on a Protein A Agarose column (PA09-R5, ABT Company). The purification was performed according to the manufacturer's instructions. Briefly, saturated ammonium sulfate was gradually added to rabbit sera at the final concertation of $33 \%$ and stirred on ice for one hour. After 25 minutes of centrifugation at $9000 \mathrm{x}$ $\mathrm{g}$, the supernatant was removed and led on the protein A column. The column was washed by PBS, and elution buffer (glycine- $\mathrm{HCl}, \mathrm{pH}=2.5$ ) was added to elute the desired protein. The eluted fraction containing the pure antibody was determined by SDS-PAGE ( $4 \%$ stacking gel and $12.5 \%$ separating gel), and the concentration of the obtained antibody was determined by the Bradford method. The eluted IgGs were immediately neutralized to physiological $\mathrm{pH}$. Molecular mass cutoff concentrators (10kDa) Amicon ultrafiltration device were exploited to concentrate the lgGs up to $1 \mathrm{mg} / \mathrm{ml}$.

\subsection{ELISA test on patients serums}

To assess the ability of the candidate vaccine proteins to interact with the antibody available in the serum of the patient (all study participants received a full explanation of the study and were obtained a written informed consent prior to their inclusion in the study), an ELISA test was designed and developed, and employed. Briefly, $1 \mu \mathrm{g}$ of each purified protein was coated onto a $96-$ well immunoplate at $37 \mathrm{C}$ overnight. Then, the serum samples from two SARS-CoV-2 patients were added to the wells. Serum from healthy people was also added to control wells. Afterward, 1/10000 dilution of anti-human IgG (HRP conjugated) was added to the wells. Finally, 50 $\mathrm{\mu l}$ of tetramethylbenzidine substrate reagent (BD Biosciences Pharmingen, CA, USA) was added to each well and incubated for $15 \mathrm{~min}$ at $37^{\circ} \mathrm{C}$. Each well gets $50 \mu \mathrm{l}$ of 2 $\mathrm{N}$ of $\mathrm{HCl}$ to stop the color development. The cut off was calculated by the following formula [30]: 
Cutoff $=X_{\text {neg }}+0.13 \bar{x}_{\text {pos }}$

Such type of assays was repeated for several samples obtained from people infected with the virus (already detected by RT- PCR). Also, a conventional rapid immuno-chromatography test was used to observe the interaction of purified antibody with the antigen-coated onto the membrane in such assays.

\subsection{Preparation of cells and virus stock}

To perform the neutralization assays, the African green monkey kidney, Vero E6 cells were employed. These cells were cultured in Dulbecco modified Eagle medium (DMEM) supplemented with $10 \%$ heatinactivated fetal bovine serum (FBS), $100 \mathrm{mg} / \mathrm{ml}$ of streptomycin, 100 units $/ \mathrm{ml}$ of penicillin $\mathrm{G}, 2 \mathrm{mM} \mathrm{L-}$ glutamine. The culture conditions were set to $5 \% \mathrm{CO}_{2}$ at $37^{\circ} \mathrm{C}$, and the cells were grown up to confluency of $70-80 \%$. The SARS-CoV-2 was obtained from COVID-19 patients and propagated in Vero E6 cells, adapting a method developed by Harcourt et al[31]. A unique biosafety level 3 (BSL-3) laboratory was used to handle the virus and the infected cell cultures. The procedures were performed following the instructions approved by Institutional Biosafety Committee (IBC).

\subsection{Cytopathic effect (CPE) based neutralization assays}

The CPE based neutralization assays were carried out in 96-well microtiter plates in triplicate. Both rabbit sera (immunized by P1, P2, and P3 antigens) and the virus stock was prepared in 1:10, 1:100, and 1:1000 dilutions. Then, $250 \mu \mathrm{l}$ of each viral dilution was incubated with each dilution of the rabbit sera for 2 hours at $37^{\circ} \mathrm{C}$. As the negative control group, $250 \mu$ l of each viral dilution was incubated for 2 hours at $37^{\circ} \mathrm{C}$ with cell culture medium without any serum. The serum-virus mixture was added on 20,000 Vero E6

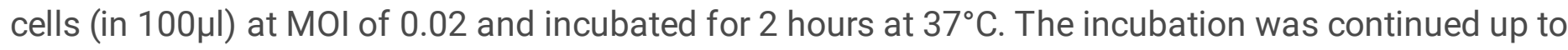
CPE appearance, which was first observed on day 5 of infection under a microscope. The definition for neutralizing antibody titer was the reciprocal of the highest serum dilution at which none of the triplicate testing wells was observed with CPE breakthrough.

\section{Results}

\subsection{Obtaining the proper sequences}

The sequence of the S protein was stored under the reference Sequence ID of YP_009724390.1. The BLAST search based on this sequence has returned numerous protein sequences with significant coverage and identity. The MSA results confirm the previous studies showing the conserved and variable regions of the S protein [32]. The variability in the RBM sequence was also evidently analyzing the MSA results. There were 17 asparagine residues predicted to be $\mathrm{N}$-glycosylated throughout the $\mathrm{S}$ sequence. The glycosylation was less condensed at the RBD region and the region connecting the S1 and S2 parts. The sequence spanning the residues $1-15$ was predicted to be a signal.

\subsection{Selection of regions for vaccine candidates}

Given the properties of the $S$ protein and considering the mechanism of $S$ protein to fuse the virus into the host cells, three areas, including the RBD (P1), fusion peptide (P2), and S1/S2 cleavage site (P3) of the 
spike protein were selected to be the candidate vaccine antigens. Their molecular weight was calculated to be 11477.82 Da, 13528.71 Da, and 25000 Da for P1, P2, and P3 antigens, respectively. The selected vaccine candidates were all predicted to be stable according to their instability index and have a high estimated half-life within mammalian cells. Analyzing the properties of these antigens, there were no asparagine residues with possible glycosylation, all three vaccine candidates were predicted to be antigens, no allergenicity effects were expected to be inclined by these vaccine candidates, and there were no regions with significant potential of toxicity throughout their sequences.

\subsection{Protein expression}

The corresponding genes for P1, P2, and P3 vaccine candidates were optimized according to the $\mathrm{E}$. coli codon usage bias, and the unwanted sequences which could affect the optimal protein expression were omitted. Gene cloning was confirmed by DNA sequencing and enzymatic digestion on the sub-cloned vector. The colony PCR confirmed that the vectors are transformed into the E.coli BL21 (DE3) host. The results of the protein expression have shown that all $\mathrm{P} 1, \mathrm{P} 2$, and $\mathrm{P} 3$ proteins are overexpressed and can travel to the expected molecular weight on SDS-PAGE gel (12 kDa for P1, $14 \mathrm{kDa}$ for P2, and $25 \mathrm{kDa}$ for P3) (Fig. 1). It has been revealed that the best condition for the expression of the proteins is $1 \mathrm{mM}$ of IPTG for 4 hours at $37^{\circ} \mathrm{C}$.

\subsection{Protein purification and Western blot analysis}

The expressed proteins for P1, P2, and P3 neutralizing antibody candidates were purified by Ni+-NTA resin-packed columns following the denaturation and renaturation procedure $(\mathrm{pH}$ change and urea alternation) and the dialyses. Running the purified protein samples on the SDS-PAGE gel indicated that the unwanted proteins moieties are removed from the samples, and the purified proteins can travel to their expected molecular weight (Fig. 2). The Western blot analysis has also confirmed the identity of the purified antigens using the anti-His tag antibody (Fig. 3).

\subsection{CD analyses}

The results of the $C D$ analyses for the P1, P2, and P3 vaccine candidates are listed in Table 1. The obtained results indicated that all three vaccine candidates folded to discernible secondary structures with different ratios. 
Table 1

CD results for the $P 1, P 2$, and $P 3$ vaccine candidates (OM Urea is the base buffer without urea).

\begin{tabular}{|c|c|c|c|c|c|c|c|c|c|}
\hline & \multirow{2}{*}{$\begin{array}{l}\text { Secondary } \\
\text { structure (\%) }\end{array}$} & \multirow[t]{2}{*}{ PBS } & \multirow{2}{*}{$\begin{array}{l}\text { OM } \\
\text { Urea }\end{array}$} & \multirow{2}{*}{$\begin{array}{l}1 \mathrm{M} \\
\text { Urea }\end{array}$} & \multirow{2}{*}{$\begin{array}{l}2 \mathrm{M} \\
\text { Urea }\end{array}$} & \multirow{2}{*}{$\begin{array}{l}4 \mathrm{M} \\
\text { Urea }\end{array}$} & \multirow{2}{*}{$\begin{array}{l}6 \mathrm{M} \\
\text { Urea }\end{array}$} & \multirow{2}{*}{$\begin{array}{l}8 \mathrm{M} \\
\text { Urea }\end{array}$} & \multirow[t]{2}{*}{ Prediction } \\
\hline & & & & & & & & & \\
\hline \multirow[t]{4}{*}{ P1 } & a-helix & 12.10 & 100.00 & 35.80 & 48.00 & 23.30 & 0.00 & 32.30 & 5.94 \\
\hline & $\beta$-sheet & 61.60 & 0.00 & 0.00 & 0.00 & 38.50 & 60.00 & 0.0 & 26.73 \\
\hline & $\beta$-turn & 26.30 & 0.00 & 64.20 & 52.00 & 28.70 & 0.00 & 41.90 & 5.94 \\
\hline & Random coil & 0.00 & 0.00 & 0.00 & 0.00 & 9.50 & 40.00 & 25.80 & 61.39 \\
\hline \multirow[t]{4}{*}{ P2 } & a-helix & 36.00 & 33.90 & 49.70 & 12.80 & 13.30 & 85.50 & 56.40 & 38.14 \\
\hline & $\beta$-sheet & 0.00 & 66.10 & 0.00 & 54.70 & 34.60 & 0.00 & 0.00 & 12.37 \\
\hline & $\beta$-turn & 64.00 & 0.00 & 50.30 & 7.50 & 31.50 & 0.00 & 43.60 & 5.15 \\
\hline & Random coil & 0.00 & 0.00 & 0.00 & 25.00 & 20.60 & 14.50 & 0.00 & 44.33 \\
\hline \multirow[t]{4}{*}{ P3 } & a-helix & 7.70 & 74.70 & 38.00 & 75.10 & 19.80 & 100.0 & 59.20 & 14.37 \\
\hline & $\beta$-sheet & 33.50 & 0.00 & 0.00 & 0.00 & 52.20 & 0.00 & 0.00 & 26.35 \\
\hline & $\beta$-turn & 36.70 & 0.00 & 62.00 & 0.00 & 21.90 & 0.00 & 40.80 & 8.98 \\
\hline & Random coil & 22.00 & 25.30 & 0.00 & 24.90 & 6.10 & 0.00 & 0.00 & 50.30 \\
\hline
\end{tabular}

\subsection{Antibody production and purification}

After performing the immunization regiment on both rabbits and mice, their serum samples were evaluated for antibody elicitation. The results of indirect ELISA tests for both animal groups indicated that antibodies were raised against P1, P2, and P3 protein candidates (Figs. 4 and 5). The results of these indirect ELISA tests confirmed the immunogenicity of the P1, P2, and P3 protein candidates within both rabbits and mice without any lethal consequences. The obtained results have also confirmed long-lasting antibody response for $\mathrm{P} 1, \mathrm{P} 2$, and $\mathrm{P} 3$ protein candidates. The obtained results showed that the antibody titer for all three rabbit groups (injected with P1, P2, and P3 proteins) remains higher than the cut off value up to 130 days after injection. Similarly, the antibody titer was higher than the cutoff value for mice groups (injected with P3, P1, P2, and a mixture of three proteins) up to 170 days after the second injection. However, a decreasing trend for antibody titer was detected in the test results of days 130 and 170 compared to the previous days. The affinity chromatography on a Protein A column managed to purify the IgG antibodies from the serum samples (Fig. 6).

\subsection{ELISA test on serum sample of COVID-19 patients}


Using the P1, P2, and $\mathrm{P} 3$ protein candidates as the capture antigen in an ELISA test would bring about data regarding the ability of these antigens to interact with actual neutralizing antibodies produced within the serums of COVID-19 patients. The obtained results indicated that P1, P2, and P3 vaccine candidates are capable of interacting with antibodies raised within the serums from COVID-19 patients (Fig. 7). A positive interaction result could be construed as functional and structural similarities between the P1, P2, and P3 vaccine candidates and their corresponding sequences within the whole virus structure.

\subsection{CPE based neutralization assays}

The neutralization assay determines the effect of neutralizing antibodies based on the observation of cell morphology. This method is reported to be the first and most frequently used neutralization assay in the SARS research [33]. The obtained results indicated that it takes 5 days for the infected Vero E6 cells to start forming visible CPE including dissociated cell patterns and that the 1:10 concentration of the rabbit serum immunized by the P1, P2, and P3 vaccine candidates could neutralize the 1:10, 1:100, and 1:1000 concentrations of viral stock. The 1:100 concentration of the rabbit serum immunized by $\mathrm{P} 1$ vaccine candidate could neutralize up to the 1:1000 concentrations of viral stock (Fig. 8).

\section{Discussion}

Employing an integrative approach, we aimed to produce a safe and inexpensive vaccine against the SARS-CoV-2. In this regard, the subunit vaccine platform is based on S protein. Previous studies on SARSCoV, MERS-CoV, and recent SARS-CoV-2 have revealed that given the critical role of S protein in the mechanism behind the virus entry into the host cells, it can be deemed as the best candidate for vaccine development efforts. Usually, the whole S protein (or its subunits) or the RBD region is used for the design of a subunit vaccines against the SARS-CoV-2. The vaccine is usually injected along with a suitable adjuvant to get the best immunization results. The Novavax subunit vaccine uses the complete $S$ glycoprotein in combination with the MATRIX M adjuvant. This vaccine is now in phase 2 clinical trials. Prior studies have reported that selecting the whole sequence of the $S$ protein could trigger unwanted immune responses. These responses could lead to inflammatory and hepatic damage or increased infection after exposure to SARS-CoV in the animal models. In light of these facts, encompassing the whole $S$ protein sequence in the designed subunit vaccine could be associated with adverse effects[3437]. Inclusion of the RBD region instead of the whole $S$ protein is offered as an alternative for anti-SARS$\mathrm{CoV}-2$ vaccine design efforts. This region has been shown to produce a higher antibody titer compared to the immunization with the whole $S$ protein. The rational underlying this observation could be the existence of immuno-dominant non-neutralizing epitopes within the sequence of the whole $S$ protein sequence. These epitopes would trigger the immune system toward themselves, and the neutralizing epitopes would remain unresponsive. Ultimately, the elicitation of sufficient titer of the neutralizing antibodies would not be accomplished [31, 38-41]. Monoclonal antibodies produced against different epitopes of the RBD region of S protein have been reported to be effective against different strains of the SARS virus isolated from patients at various stages of the disease. These antibodies are predicted to provoke neutralizing effects on SARS-CoV-2. Moreover, unlike inactivated vaccines based on whole S 
protein, RBD based immunization was not associated with Antibody-dependent Enhancement (ADE) or other detrimental immune responses [21].

In silico studies, have garnered a lot of attention in the design of subunit vaccines Kar et al. have developed a multi-epitope vaccine based on the selected epitopes from the S glycoprotein of the SARSCoV-2 [31] In a similar approach, another multi-epitope vaccine was designed and assessed based on the S protein epitopes of the SARS-CoV-2 [42]. The promising results of these studies have convinced us to use a combination of in silico studies and information from previous studies to design three vaccine candidate antigens. The extent of conservancy, glycosylation, toxicity, antigenicity, and immunogenicity were the criteria considered for the vaccine design and selection.

Folding to a native-like structure is highly important for the designed recombinant proteins to invoke proper immune responses. Since the protein purification was performed in urea containing solutions, the secondary structure of the protein would be affected. Urea can denature the protein structures by direct and indirect mechanisms. Regarding the direct mechanism, the urea could bind directly to the charged and polar side chains of the protein via hydrogen bonding and other electrostatic interactions, it could bind directly to amino acids through van der Waals attractions, and it could bind the side chains via a combination of these two methods. Stronger dispersion interaction of the urea with protein than water, supports the direct interaction mechanism [43]. This is while in the indirect mechanism, urea leads to the easier dissolution of hydrophobic protein groups by disrupting the structure of water. It has been reported that with a gradual increase in urea concentration from 6-10 $\mathrm{M}$, the beta sheets will be destroyed, the alpha helixes will remain stable and may even increase, and the random coils will remain intact $[44,45]$. The obtained CD results for the P1, P2, and P3 vaccine candidates have confirmed these structural changes during urea removal. This could be construed as protein refolding to its native structure upon urea removal.

Yuxian et al. have also used a recombinant fusion protein (RBD-Fc) as an immunogen to immunize rabbits [46]. The RBD region was selected due to its functional role in virus entry and inclusion of neutralizing epitope. The RBD-Fc antigen induced robust antibody responses and ultimately prevented the SARS-CoV infection in the diluted serum [31]. Similar results were reported in a study conducted by Du et al. in mice [47]. In line with the results of previous studies, our results have shown that the injection of purified proteins of different parts of $S$ protein has led to a significant increase in the neutralizing antibody titer of the immunized animals. This antibody titer remained high for 130 and 170 days after the initial injections for rabbit and mouse groups, respectively. This property indicates the elicitation of a robust immune response. The ELISA test against the serums of the COVID-19 patients confirmed the ability of the elicited polyclonal response to detecting the $S$ protein within the serum of COVID-19 patients.

To have an efficient immune response against the SARS-CoV-2, the elicited antibodies should exhibit the ability to neutralize the viral particle. The Virus Neutralization Test (VNT) method is a susceptible and specific test to check for the presence of neutralizing antibodies against the target virus. This method is 
also practiced as the gold standard method to analyze the presence of neutralizing antibodies against SARS-CoV-2. Previous studies have demonstrated that the $\mathrm{S}$ protein components such as S2, S1, and especially RBD hold a profound potential for production of neutralizing antibodies. These antibodies can block the virus binding to the ACE2 receptor and its membrane fusion[31]. Our VNT results have also confirmed that the elicited antibodies against the designed proteins are highly persistent and capable of neutralizing the cultured live virus. This property could be construed as the highly promising potential of these proteins as vaccine candidates.

However, for further analyses, these recombinant proteins should be injected into a higher mammal like Macaque rhesus monkey to assess their efficiency in higher species. Currently, we are conducting this experiment on the Macaque rhesus monkey. The available data (although auspicious) are not sufficient for definitive conclusions, and more time and experiments are needed to show the performance of the designed recombinant proteins in higher species. However, taking the results into account, these antigens appear to be compelling vaccine candidates against the SARS-CoV-2 owing to their ability to develop potent neutralizing antibodies, long-term immunity in animals, and no apparent side effects. Future studies will disclose the true potential of these subunit vaccine candidates for vaccination against COVID-19.

\section{Declarations}

Acknowledgment: We gratefully thank the National Institute of Genetic Engineering and Biotechnology for the financial support.

\section{References}

1. Zhang, J., et al., Recommended psychological crisis intervention response to the 2019 novel coronavirus pneumonia outbreak in China: a model of West China Hospital. Precision Clinical Medicine, 2020. 3(1): p. 3-8.

2. Singhal, T., A review of coronavirus disease-2019 (COVID-19). The Indian Journal of Pediatrics, 2020: p. 1-6.

3. Funk, C.D., C. Laferrière, and A. Ardakani, A snapshot of the global race for vaccines targeting SARSCoV-2 and the COVID-19 pandemic. Frontiers in pharmacology, 2020. 11: p. 937.

4. Zhang, W., et al., Molecular and serological investigation of 2019-nCoV infected patients: implication of multiple shedding routes. Emerging microbes \& infections, 2020. 9(1): p. 386-389.

5. Speiser, D.E. and M.F. Bachmann, COVID-19: Mechanisms of Vaccination and Immunity. Vaccines (Basel), 2020. 8(3).

6. Ralph, R., et al., 2019-nCoV (Wuhan virus), a novel Coronavirus: human-to-human transmission, travel-related cases, and vaccine readiness. The Journal of Infection in Developing Countries, 2020. 14(01): p. 3-17. 
7. Lu, R., et al., Genomic characterisation and epidemiology of 2019 novel coronavirus: implications for virus origins and receptor binding. The Lancet, 2020. 395(10224): p. 565-574.

8. Guo, Y.-R., et al., The origin, transmission and clinical therapies on coronavirus disease 2019 (COVID19) outbreak-an update on the status. Military Medical Research, 2020. 7(1): p. 1-10.

9. Nasr Esfahani, B.N., A.M. Ahadi, and S. Shalibeik, A Review of Novel Coronavirus, Severe Acute Respiratory Syndrome Coronavirus 2 (SARS-CoV-2). Iranian Journal of Medical Microbiology, 2020. 14(2): p. 154-161.

10. Wu, F., et al., A new coronavirus associated with human respiratory disease in China. Nature, 2020. 579(7798): p. 265-269.

11. Cui, J., F. Li, and Z.-L. Shi, Origin and evolution of pathogenic coronaviruses. Nature Reviews Microbiology, 2019. 17(3): p. 181-192.

12. Wu, A., et al., Genome composition and divergence of the novel coronavirus (2019-nCoV) originating in China. Cell host \& microbe, 2020.

13. Hoffmann, M., et al., The novel coronavirus 2019 (2019-nCoV) uses the SARS-coronavirus receptor ACE2 and the cellular protease TMPRSS2 for entry into target cells. BioRxiv, 2020.

14. Zhang, H., et al., The digestive system is a potential route of 2019-nCov infection: a bioinformatics analysis based on single-cell transcriptomes. BioRxiv, 2020.

15. Jia, H.P., et al., ACE2 receptor expression and severe acute respiratory syndrome coronavirus infection depend on differentiation of human airway epithelia. Journal of virology, 2005. 79(23): p. 14614-14621.

16. Wan, Y., et al., Receptor recognition by the novel coronavirus from Wuhan: an analysis based on decade-long structural studies of SARS coronavirus. Journal of virology, 2020. 94(7).

17. Tortorici, M.A. and D. Veesler, Structural insights into coronavirus entry, in Advances in virus research. 2019, Elsevier. p. 93-116.

18. Weissenhorn, W., et al., Structural basis for membrane fusion by enveloped viruses. Molecular membrane biology, 1999. 16(1): p. 3-9.

19. Wrapp, D., et al., Cryo-EM structure of the 2019-nCoV spike in the prefusion conformation. Science, 2020. 367(6483): p. 1260-1263.

20. Xia, S., et al., Fusion mechanism of 2019-nCoV and fusion inhibitors targeting HR1 domain in spike protein. Cellular \& molecular immunology, 2020: p. 1-3.

21. Yu, F., et al., Measures for diagnosing and treating infections by a novel coronavirus responsible for a pneumonia outbreak originating in Wuhan, China. Microbes and infection, 2020.

22. Zhang, N., S. Jiang, and L. Du, Current advancements and potential strategies in the development of MERS-CoV vaccines. Expert Rev Vaccines, 2014. 13(6): p. 761-74.

23. Jiang, S., L. Du, and Z. Shi, An emerging coronavirus causing pneumonia outbreak in Wuhan, China: calling for developing therapeutic and prophylactic strategies. Emerg Microbes Infect, 2020. 9(1): p. 275-277. 
24. Pillay, T.S., Gene of the month, the 2019-nCoV/SARS-CoV-2 novel coronavirus spike protein. Journal of Clinical Pathology, 2020.

25. Du, L., et al., , The spike protein of SARS-CoV-a target for vaccine and therapeutic development. Nature Reviews Microbiology, 2009. 7(3): p. 226-236.

26. Wong, S.K., et al., , A 193-amino acid fragment of the SARS coronavirus S protein efficiently binds angiotensin-converting enzyme 2. Journal of Biological Chemistry, 2004. 279(5): p. 3197-3201.27.

27. Li, $\mathrm{F}$. , et al., , Conformational states of the severe acute respiratory syndrome coronavirus spike protein ectodomain. Journal of virology, 2006. 80(14): p. 6794-6800..

28. Du, L., et al., The spike protein of SARS-CoV-a target for vaccine and therapeutic development. Nature Reviews Microbiology, 2009. 7(3): p. 226-236.

29. Mard-Soltani, M., et al., Eliciting an antibody response against a recombinant TSH containing fusion protein. Journal of Immunoassay and Immunochemistry, 2017. 38(3): p. 257-270.

30. Lardeux, F., G. Torrico, and C. , Aliaga, Calculation of the ELISA's cut-off based on the change-point analysis method for detection of Trypanosoma cruzi infection in Bolivian dogs in the absence of controls. Mem Inst Oswaldo Cruz, 2016. 111(8): p. 501-4.

31. Harcourt, J., et al.,, Severe Acute Respiratory Syndrome Coronavirus 2 from Patient with Coronavirus Disease, United States. Emerg Infect Dis, 2020. 26(6): p. 1266-1273.

32. Jaimes, J.A., et al., Phylogenetic Analysis and Structural Modeling of SARS-CoV-2 Spike Protein Reveals an Evolutionary Distinct and Proteolytically Sensitive Activation Loop. Journal of molecular biology, 2020. 432(10): p. 3309-3325.

33. Wang, S., et al., Identification of two neutralizing regions on the severe acute respiratory syndrome coronavirus spike glycoprotein produced from the mammalian expression system. Journal of virology, 2005. 79(3): p. 1906-1910.

34. Czub, M., et al., Evaluation of modified vaccinia virus Ankara based recombinant SARS vaccine in ferrets. Vaccine, 2005. 23(17-18): p. 2273-2279.

35. Weingartl, $\mathrm{H}$., et al., Immunization with modified vaccinia virus Ankara-based recombinant vaccine against severe acute respiratory syndrome is associated with enhanced hepatitis in ferrets. Journal of virology, 2004. 78(22): p. 12672-12676.

36. Honda-Okubo, Y., et al., Severe acute respiratory syndrome-associated coronavirus vaccines formulated with delta inulin adjuvants provide enhanced protection while ameliorating lung eosinophilic immunopathology. Journal of virology, 2015. 89(6): p. 2995-3007.

37. Sekimukai, H., et al., Gold nanoparticle-adjuvanted S protein induces a strong antigen-specific IgG response against severe acute respiratory syndrome-related coronavirus infection, but fails to induce protective antibodies and limit eosinophilic infiltration in lungs. Microbiology and Immunology, 2020. 64(1): p. 33-51.

38. Pancino, G., et al., Conserved framework of envelope glycoproteins among lentiviruses, in Simian Immunodeficiency Virus. 1994, Springer. p. 77-105. 
39. Geisbert, T.W., et al., Evidence against an important role for infectivity-enhancing antibodies in Ebola virus infections. Virology, 2002. 293(1): p. 15-19.

40. Takada, A. and Y. Kawaoka, Antibody-dependent enhancement of viral infection: molecular mechanisms and in vivo implications. Reviews in medical virology, 2003. 13(6): p. 387-398.

41. Takada, A., et al., Infectivity-enhancing antibodies to Ebola virus glycoprotein. Journal of virology, 2001. 75(5): p. 2324-2330.

42. Singh, A., et al., Designing a multi-epitope peptide-based vaccine against SARS-CoV-2. bioRxiv, 2020.

43. Hua, L., et al., Urea denaturation by stronger dispersion interactions with proteins than water implies a 2-stage unfolding. Proceedings of the National Academy of Sciences, 2008. 105(44): p. 1692816933.

44. Rho, Y., et al., Chemically Denatured Structures of Porcine Pepsin using Small-Angle X-ray Scattering. Polymers, 2019. 11(12): p. 2104.

45. Bennion, B.J. and V. Daggett, The molecular basis for the chemical denaturation of proteins by urea. Proceedings of the National Academy of Sciences, 2003. 100(9): p. 5142-5147.

46. He, Y., et al., Receptor-binding domain of SARS-CoV spike protein induces highly potent neutralizing antibodies: implication for developing subunit vaccine. Biochemical and biophysical research communications, 2004. 324(2): p. 773-781.

47. Du, L., et al., Receptor-binding domain of SARS-CoV spike protein induces long-term protective immunity in an animal model. Vaccine, 2007. 15(25): p. 2832-2838.

\section{Figures}

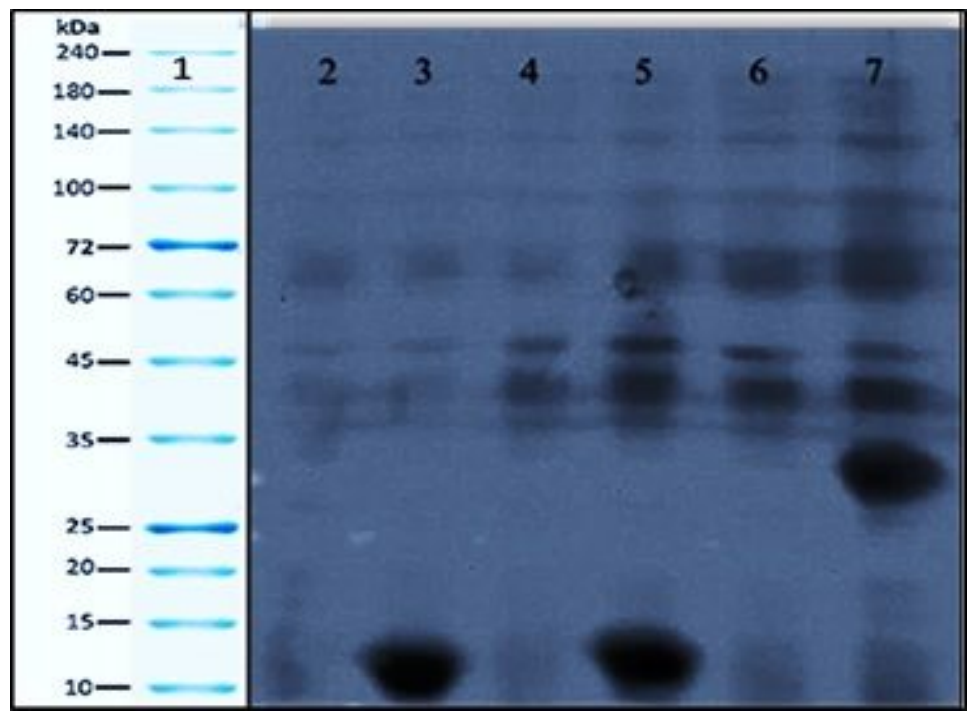

\section{Figure 1}

SDS-PAGE analyses the protein expression (Lane 1. Protein ladder, Lane 2. P1 before expression, Lane 3. P1 after expression, Lane 4. P2 before expression, Lane5. P2 after expression, Lane6. P3 before 
expression, Lane7. P3 after expression).
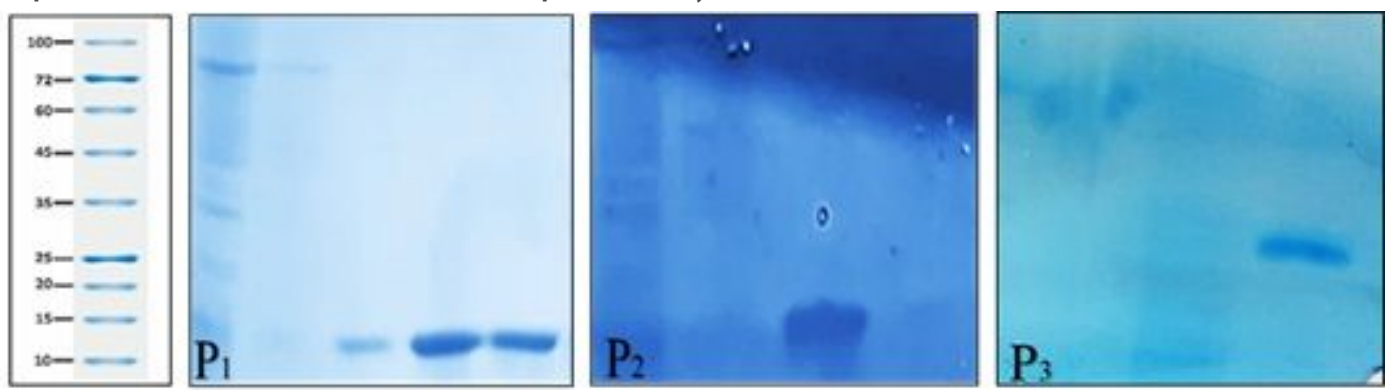

\section{Figure 2}

Protein purification. Different fractions of purified P1, P2, and P3 proteins on SDS-PAGE are shown. On the left is the Molecular weight marker.

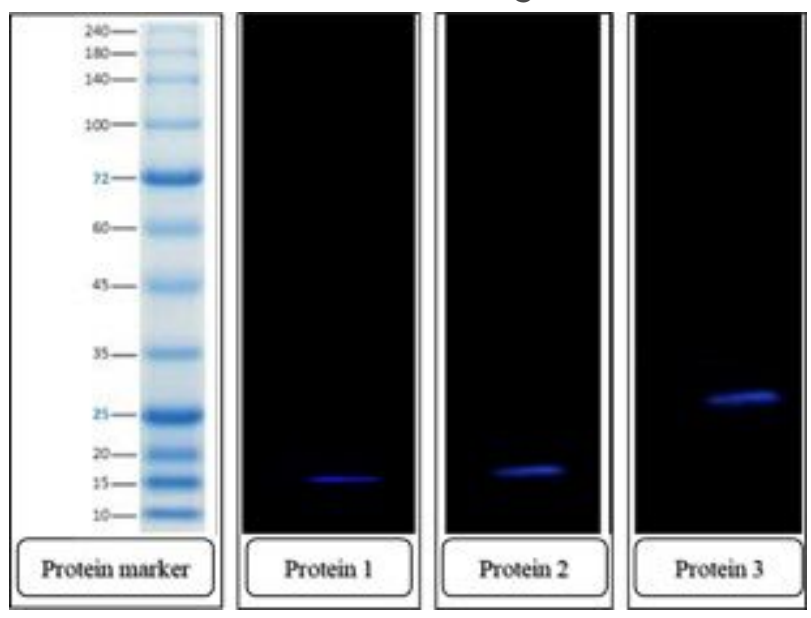

\section{Figure 3}

Protein expression confirmation by Western blotting. The blue bands are the expressed proteins. On the left is the Molecular weight marker.
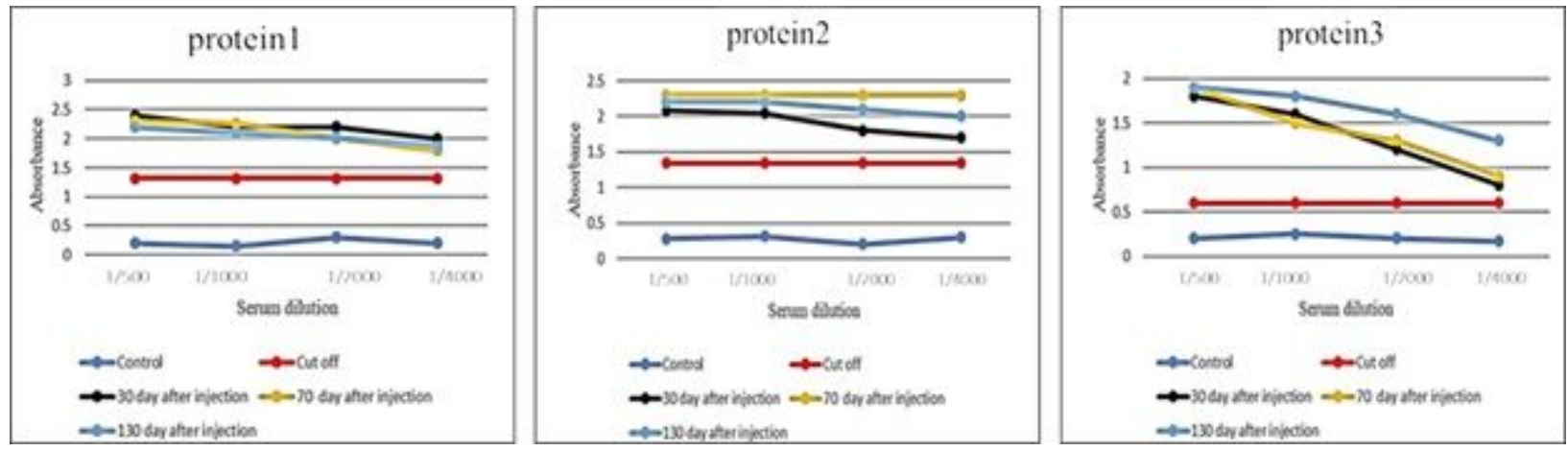

\section{Figure 4}

Serum antibody titer for rabbits immunized with $\mathrm{P} 1, \mathrm{P} 2$, and P3 proteins compared to the control group. 

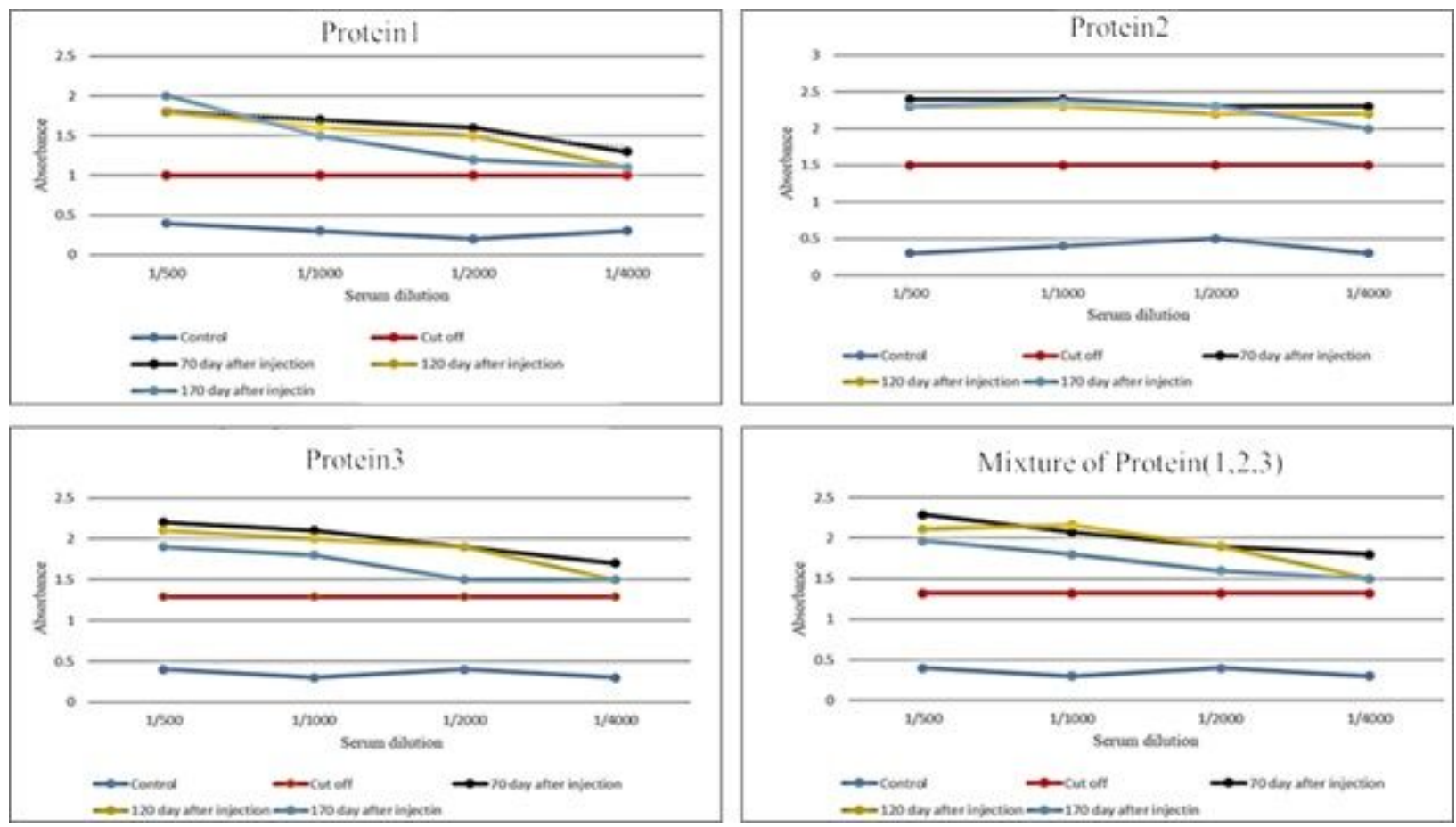

Figure 5

The serum antibody titer for mice groups immunized with P1, P2, P3, and a mixture of three proteins compared to the control group.

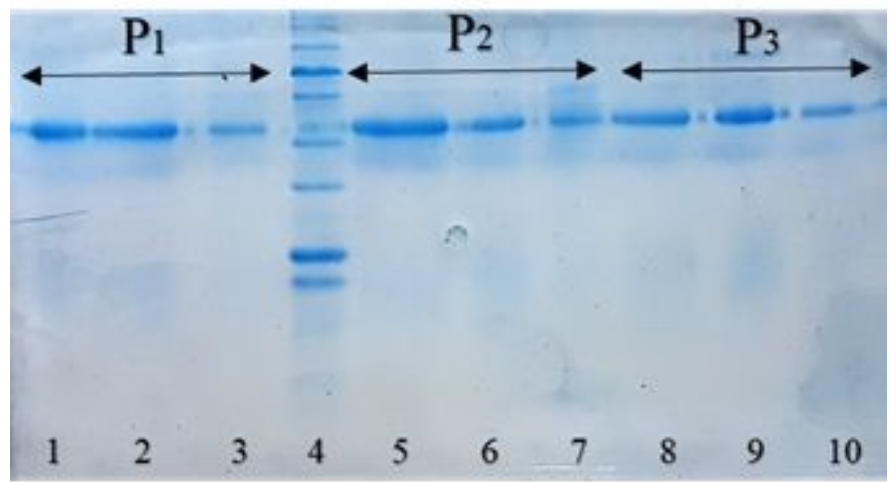

Figure 6

The fractions of antibody elution from the protein A column (lanes 1-3: elusion fractions for P1, lane 4: molecular weight, lanes 5-7: elusion fractions for P2, lanes 8-10: elusion fractions for P3). 


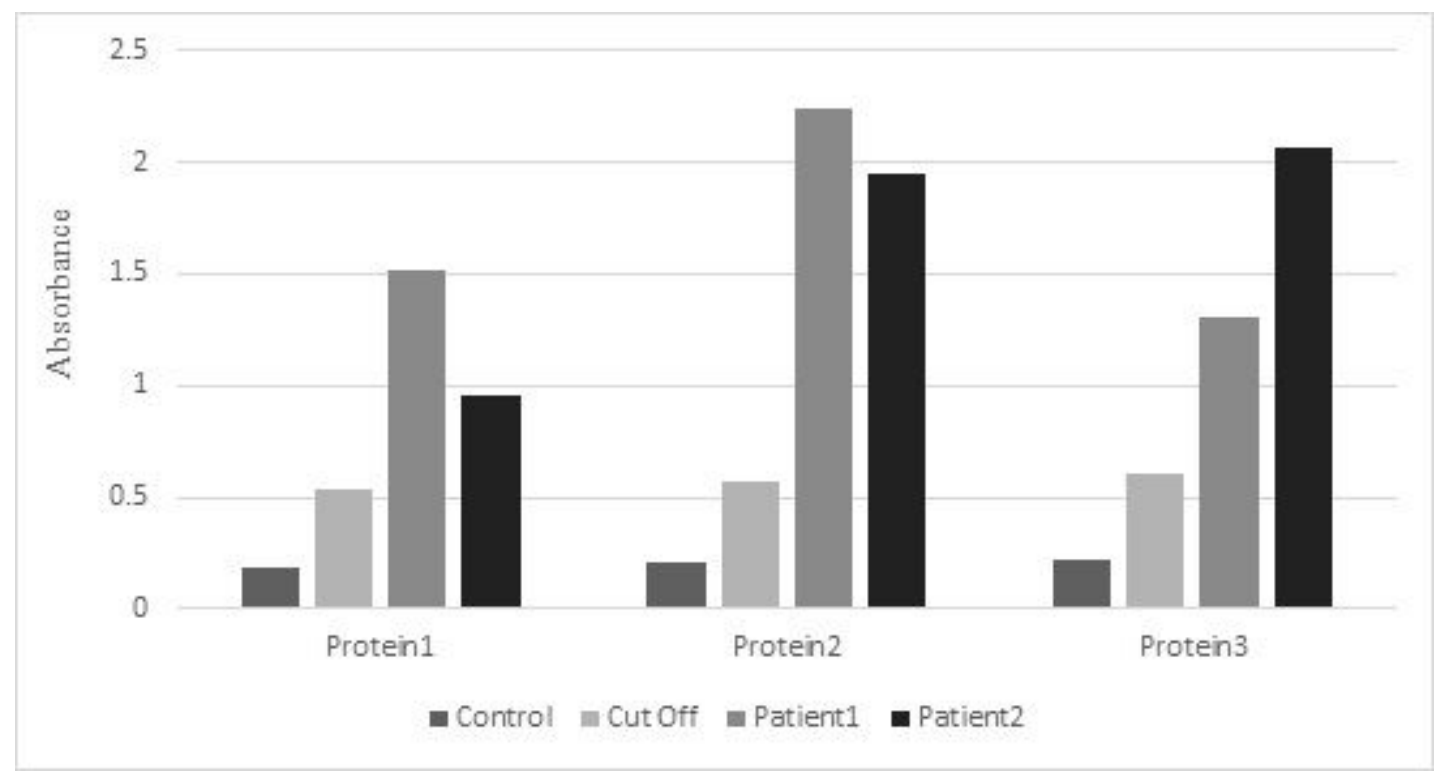

Figure 7

The ELISA results for Anti-SARS-CoV-2 antibody detection in the serum of COVID-19 patients using the $\mathrm{P} 1, \mathrm{P} 2$, and P3 proteins as capture antigens compared to the healthy subject (several other patient samples were used, the results are not shown here ).
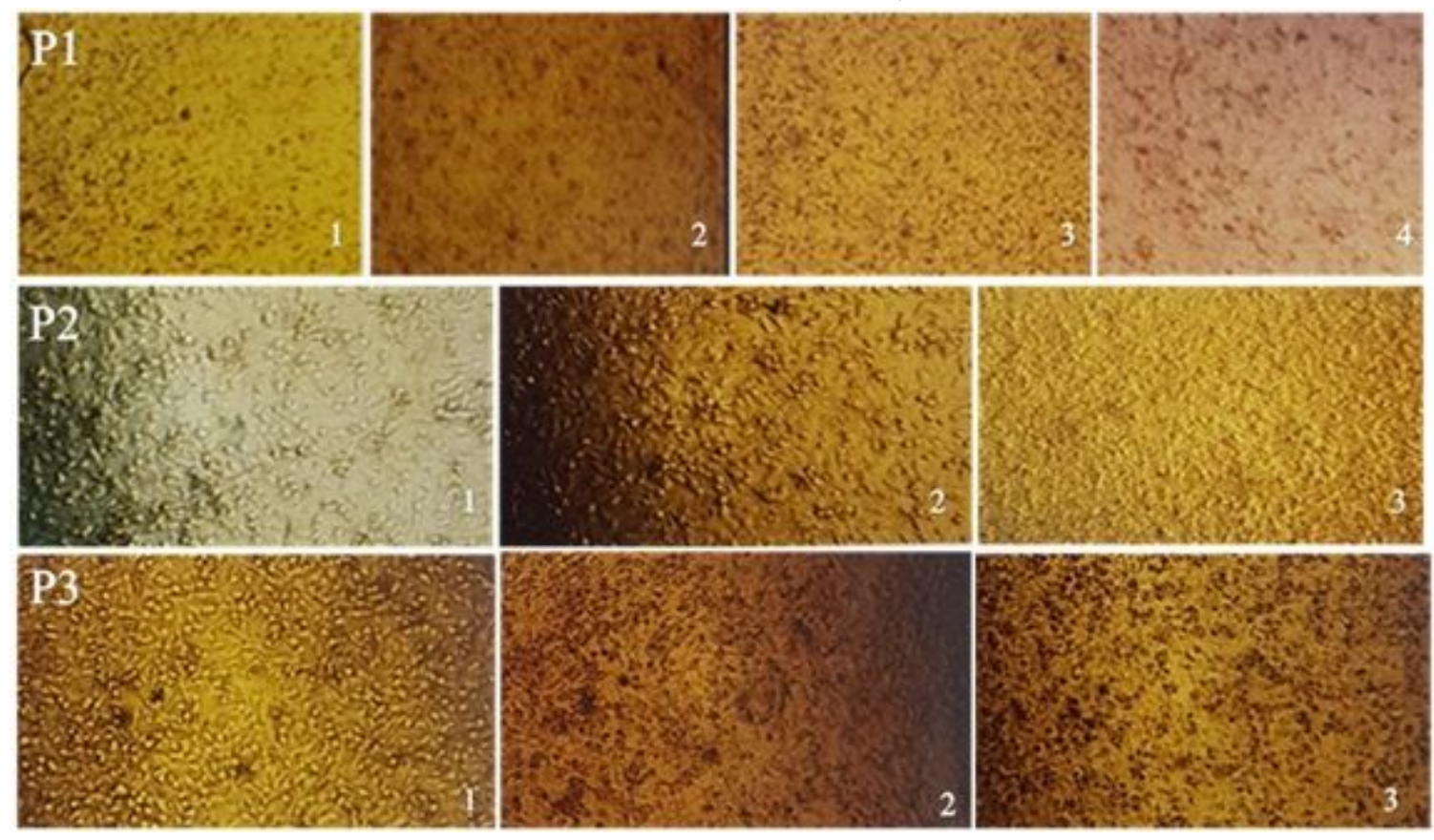

\section{Figure 8}

Different concentrations of virus neutralization via the antibodies produced by $\mathrm{P} 1, \mathrm{P} 2$, and $\mathrm{P} 3$ vaccine candidates ( 1 : is the $1: 10$ serum and 1:10 virus delusions, 2 : is the 1:10 serum and 1:100 virus delusions, 3 : is the $1: 10$ serum and 1:1000 virus delusions, 4 : is the $1: 100$ serum and 1:1000 virus delusions) 